\title{
Influence des successions culturales sur les maladies du pied et des racines du blé d'hiver
}

\author{
N Colbach ${ }^{1 *}, \mathrm{P}$ Lucas ${ }^{2}, \mathrm{~N}$ Cavelier ${ }^{1}$ \\ ${ }^{1}$ INRA, SRIV; \\ ${ }^{2}$ INRA, station de pathologie végétale, BP 29, F35650 Le Rheu, France
}

(Reçu le 29 avril 1994; accepté le 28 octobre 1994)

\begin{abstract}
Résumé - Les conséquences de différentes successions culturales sur les maladies du pied et des racines ont été étudiées sur une culture de blé d'hiver conduite de manière homogène. Les niveaux d'infestation de piétin-verse (Pseudocercosporella herpotrichoides), piétin-échaudage (Gaeumannomyces graminis var triticl) et rhizoctone (Rhizoctonia cerealis) ont été notés à la floraison sur tige et racines. Les résultats obtenus ont permis de proposer un modèle simple de l'effet du précédent et de l'antéprécédent sur les 3 maladies, basé sur le classement des risques des précédents culturaux en 3 groupes : hôte, culture amplificatrice et non-hôte pour le piétin-échaudage et le rhizoctone, hôte fort/faible, culture amplificatrice et non-hôte pour le piétin-verse. Alors que les cultures hôtes tendent à augmenter le risque et les non-hôtes à le diminuer, les cultures du groupe des amplificatrices (maïs, ray-grass...) n'entraînent un risque que si elles sont associées dans le temps à des cultures hôtes.
\end{abstract}

blé d'hiver / Pseudocercosporella herpotrichoides / Gaeumannomyces graminis / Rhizoctonia cerealis / succession culturale / capacité d'hôtes des cultures / modèles de risque parasitaire

Summary - Influence of crop succession on foot and root diseases of wheat. The consequences of different crop successions on foot and root diseases were analysed on a subsequent winter wheat crop managed identically, regardless of crop succession. At flowering, tillers and roots were assessed for eyespot (Pseudocercosporella herpotrichoides), take-all (Gaeumannomyces graminis var tritici) and sharp eyespot (Rhizoctonia cerealis). As a consequence, we can propose a simple model of the effect of the preceding crop and the 1 before that on the 3 diseases. This model is based on a crop classification into 3 groups: host, amplifying crop and non-host for take-all and sharp eyespot; and 'strong/weak' host, amplifying crop and non-host for eyespot. Whereas host crops tend to increase and non-host crops to decrease disease risk, amplifying crops (maize, ryegrass, etc) can only increase risk if they are associated with host crops.

winter wheat / Pseudocercosporella herpotrichoides / Gaeumannomyces graminis / Rhizoctonia cerealis / crop succession / host capacity of crops / disease risk model

* Correspondance et tirés à part : INRA, laboratoire d'agronomie, F78850 Thiverval-Grignon, France 


\section{INTRODUCTION}

Les agents pathogènes majeurs du complexe parasitaire de la base des tiges et des racines du blé sont Pseudocercosporella herpotrichoides (Fron) Deighton, agent du piétin-verse, Rhizoctonia cerealis Van der Hoeven, agent du rhizoctone, et Gaeumannomyces graminis (Sacc) von Arx et Olivier var tritici (Walker), agent du piétin-échaudage. Les 2 premiers sont responsables de nécroses sur gaines et plus tard, à la base des tiges de blé, tandis que le dernier provoque des nécroses noires sur les racines séminales et adventives.

Comme les agents pathogènes responsables de ces maladies se conservent, en absence de plantes hôtes, principalement sur des débris d'anciennes cultures, la succession culturale semble être un élément majeur du système de culture comme le montrent de nombreux travaux tant en France (Huet, 1986) qu'en Allemagne (Steinbrenner et Höflich, 1984 ; Groll et Luzi, 1991), en Tchécoslovaquie (Sekerkova et al, 1982), en Grande-Bretagne (Prew, 1981 ; Polley et Thomas, 1991), au Danemark (Bodker et al, 1990 ; Schulz et al, 1990). Des modèles de classement de successions en fonction de leur risque de maladies ont même été élaborés pour le piétin-verse (Steinbrenner et Obenauf, 1986 ; Groll et Luzi, 1991) et pour le piétin-échaudage (Steinbrenner et Obenauf, 1986 ; Steinbrenner et al, 1990).

Cependant, ces modèles qui indiquent uniquement un niveau d'infection moyen pour les 4 combinaisons d'antéprécédent/précédent, hôte/hôte, non-hôte/hôte, hôte/non-hôte, non-hôte/non-hôte, ne rendent pas compte des différences de sensibilité existant entre les différents non-hôtes, comme le montre l'exemple du maïs qui semble augmenter le risque de piétin-échaudage (Lucas et al, 1989), ou du colza qui pourrait favoriser aussi bien le piétin-échaudage que le piétin-verse (Lange-de la Camp et Naumann, 1978). D'autre part, aussi bien ces modèles que la plupart des autres résultats proviennent d'enquêtes où la date de semis de la céréale, conformément aux pratiques agricoles, varie en fonction de la culture précédente. Puisqu'un semis précoce est généralement à l'origine d'une augmentation de risque parasitaire, il y a donc risque de confusion des effets de la succession et de la date de semis.

En ce qui concerne le rhizoctone, non seulement il n'y a pas de résultats existants, mais en outre son étude est compliquée par l'existence d'interactions avec le piétin-verse (Reinecke et al, 1979 ; Van der Hoeven et Bollen, 1980 ; Cavelier et al, 1985).

Ce travail a comme objectif l'étude de l'influence de la succession culturale sur les maladies majeures du complexe parasitaire avec prise en compte des interactions entre champignons afin de i) classer les cultures en fonction de leur influence sur le développement de ces maladies, et de ii) créer des modèles permettant de classer les successions culturales en fonction de la nature et de la fréquence des cultures présentes. Nous utiliserons pour cela des essais comprenant un nombre important de successions qui seront suivis d'un blé conduit de manière uniforme, quel que soit le précédent, et extériorisant ainsi uniquement les effets de la succession culturale.

\section{MATÉRIEL ET MÉTHODES}

\section{Traitements expérimentaux}

Onze successions différentes (tableau I), combinant céréales à paille et autres cultures, ont été mises en

Tableau I. Successions culturales présentes dans l'essai.

\begin{tabular}{|c|c|c|c|c|c|c|c|c|c|c|c|}
\hline 0 & 1 & 2 & 3 & 4 & 5 & 6 & 7 & 8 & 9 & 10 & 77 \\
\hline & & -- & - & - & - & 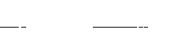 & —. & - & & $-\cdots \cdots$ & -1 \\
\hline & Blé & & Blé & & & & Blé & & & Blé & Blé \\
\hline 18 & $\mathrm{~B}$ & & r & ass & To & ta & & & & & \\
\hline & UZ & & & & & & & & & & $\pi$ \\
\hline$\pi$ & & & & & & & M & So & & $s$ & Co \\
\hline $7 / 7$ & Luzerne & Tou & Blé & & & & $\mathrm{B}$ & Blé & & Maïs & Ble \\
\hline & & & & & & & Maïs & Sor & & is & Pois \\
\hline & & Ray & & orm & & & $B$ & & & is & 1 \\
\hline & rgho & Orge hiver & Izerne & zerne & Blé & Maïs & Maïs & Sorgho & Sorgho & Maïs & Colz \\
\hline
\end{tabular}

a Orge de printemps. 
place sur une période de 12 ans, en région toulousaine (INRA, Toulouse-Auzeville) dans le cadre d'un essai de longue durée ayant pour but d'étudier divers systèmes de culture. À la 13e année (1981), un blé d'hiver (variété Top, sensible aux 3 parasites étudiés) a été implanté sur chaque parcelle et conduit de manière uniforme. Le travail du sol a cependant été adapté en fonction des résidus de cultures et la dose d'engrais azotés en fonction des reliquats azotés laissés par le précédent (tableau II), aboutissant ainsi à une quantité d'azote disponible égale dans tous les cas, correspondant à un objectif de rendement de 70 qx/ha (méthode du bilan prévisionnel, Rémy et Hébert, 1977). Les autres techniques culturales (date de semis, densité de semis...) sont identiques d'une parcelle à l'autre. Le semis a été effectué les 3 et 4 novembre, en lignes, à une densité de 350 grains par $\mathrm{m}^{2}$ et une profondeur de $2 \mathrm{~cm}$.

Le dispositif a été mis en place sur un sol alluvial de texture argilo-limoneuse à limono-argileuse. Une succession culturale donnée est suivie d'un blé irrigué et d'un blé non-irrigué, la première combinaison ayant été répétée généralement 2 à 3 fois, la deuxième 4 à 5 fois. L'irrigation a été faite en 2 apports : le premier de $40 \mathrm{~mm}$ le 23 avril au stade 7 du blé ; le deuxième de $50 \mathrm{~mm}$ le 13 mai au stade 10,1 (échelle de Feekes). Aucun traitement fongicide n'a été appliqué.
Le climat habituel de Toulouse est plutôt chaud et sec (tableau III) avec une température moyenne annuelle de $12,9^{\circ} \mathrm{C}$ et une pluviosité moyenne de $650 \mathrm{~mm} / \mathrm{an}$. Ce ne sont pas des conditions idéales pour le piétin-verse et le piétin-échaudage qui préfèrent des conditions douces et humides (Garrett, 1934 ; Ponchet, 1959 ; Schrödter et Fehrmann, 1971a, b; Asher et Shipton, 1981), mais l'année 1981-1982 était plus favorable à leur développement (tableau III). La pluviosité hivernale a été supérieure à la moyenne et accompagnée de températures douces, mais suivie d'une très forte sécheresse en avril, mai et juin.

\section{Méthodes d'études}

\section{Principe}

Dans un premier temps, nous comparons les 11 successions culturales par analyse de variance. La confrontation, entre analyse de variance et bibliographie, nous conduira à écrire un modèle de l'effet du type de précédent et d'antéprécédent sur les maladies. Nous appliquerons ce modèle à nos données dans un deuxième temps.

Tableau II. Adaptations des itinéraires techniques aux précédents.

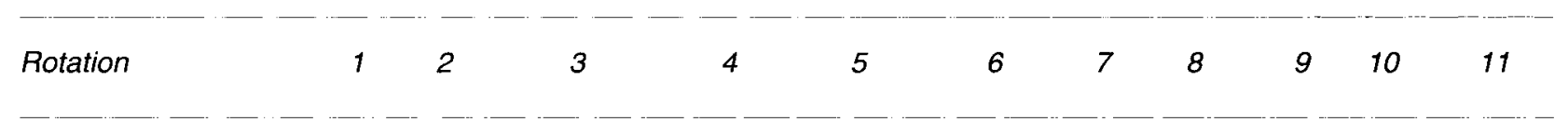

Précédent

Blé Luzerne Orge d'hiver Ray-grassTournesol Orge print Maïs Sorgho Sorgho Maïs Pois

Dose d'engrais azotésa

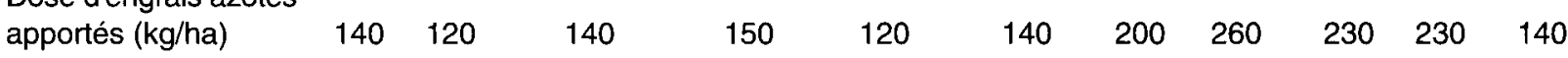

Travail du sol

Herse

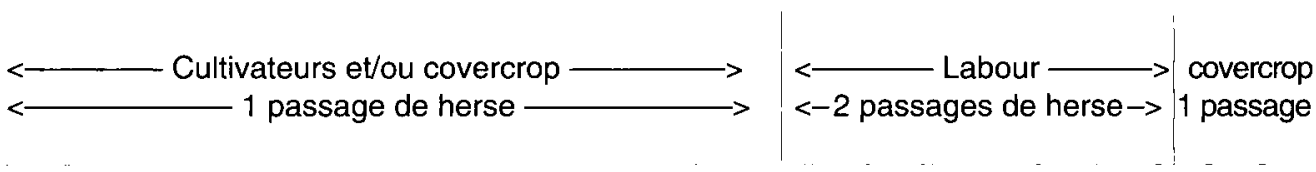

a Les engrais azotés sont apportés sous forme d'ammonitrate. La dose totale d'azote correspond à un objectif de rendement de 70qx/ha (méthode Rémy-Herbert).

Tableau III. Données climatiques moyennes et de l'année de culture.

Température $\left.{ }^{\circ} \mathrm{C}\right)$

Moyenne sur 30 ans

Mensuelle en 81-82 18,5

13,3

14,7

\section{8,3 \\ 8,5}

5,4
7,7

4,6

5,7

$9,3 \quad 11,5$

8,4

$8,2 \quad 11,5$

$14,9 \quad 18,7 \quad 20,8$

14,920

$22,7 \quad 19,6$

\section{Pluviométrie $(\mathrm{mm})$}

$\begin{array}{lllllllllllll}\text { Moyenne sur } 30 \text { ans } & 64 & 45 & 51 & 67 & 49 & 46 & 53 & 50 & 75 & 61 & 44 & 54\end{array}$

170,5

49,5

74,5

$\begin{array}{llllll}53 & 50 & 75 & 61 & 44 & 54 \\ 63 & 10,5 & 41 & 40 & 62,5 & 78,5\end{array}$




\section{Mesures pathologiques}

La variable à expliquer est le niveau d'infection parasitaire dont l'appréciation se fait par notation visuelle des lésions à la floraison. Pour chaque maladie et chaque parcelle, nous avons noté 25 plantes prises au hasard dans chaque parcelle.

Pour le piétin-verse et le rhizoctone, nous avons uniquement noté la présence de lésions sur la plante. Dans le cas du piétin-échaudage, les plantes sont en plus réparties dans 5 catégories : $0:$ plante saine ; 1 : plante dont la proportion du système racinaire nécrosé (SRN) ne dépasse pas 25\%; 2 : SRN de 26 à $50 \% ; 3$ : SRN de 51 à $75 \% ; 4$ : SRN supérieur à $75 \%$.

Une note de gravité est calculée en utilisant la formule où pei est le nombre de plantes dans la classe $i$ et est pondéré par la valeur centrale de la classe du système racinaire nécrosé :

$$
\begin{aligned}
\text { gravité } & =\left(0,125^{\star} \text { pe } 1+0,375^{\star} \text { pe } 2+0,625^{\star} \text { pe } 3\right. \\
& \left.+0,875^{\star} \text { pe } 4\right) /(\text { pe } 1+\text { pe } 2+\text { pe } 3+\text { pe } 4)
\end{aligned}
$$

Cette pondération permet d'obtenir une variable de sortie réflétant la moyenne des pourcentages de système racinaire nécrosé par plante pour chaque parcelle.

\section{Méthodes statistiques}

Dans la première partie, la méthode utilisée est l'analyse de variance, suivie d'une comparaison de moyennes 2 à 2 (test de la plus petite différence significative), si le facteur testé dans l'analyse de variance est significatif. Cette comparaison permettra de classer les successions culturales et de séparer les cultures présentes, avec l'aide des informations issues de la bibliographie, en plusieurs groupes suivant leur sensibilité et leur influence sur les maladies.

Dans une deuxième partie, ce classement de cultures trouvera son expression dans la construction, pour chaque pathogène, d'un modèle linéaire déterminé par ajustement aux moindres carrés, reliant le niveau d'infestion du blé à plusieurs facteurs : la présence ou absence d'irrigation et la nature des cultures en précédent et en antéprécédent. Nous avons également introduit les covariables : nombre de plantes par $m^{2}$ et quantité d'azote absorbé, pour prendre en compte d'éventuelles variations du peuplement qui pourraient influencer les maladies. Dans le cas du rhizoctone, le niveau d'infection par le piétin-verse est également rajouté comme covariable.

II s'agit donc d'un essai non-entièrement croisé dans lequel certaines combinaisons de précédents et antéprécédents ne sont pas représentées. L'effet antéprécédent ne pourra donc pas être étudié indépendamment de l'effet précédent et les modèles linéaires testés comprendront un effet antéprécédent hiérarchisé sous l'effet précédent et pas d'effet principal pour l'antéprécédent. Le modèle initial testé est donc de la forme : maladie $=$ effet précédent + effet antéprécédent hiérarchisé sous l'effet précédent + effet irrigation $+a^{*}$ plantes par $m^{2}+b^{*}$ quantité d'azote absorbé $\left(+c^{*}\right.$ piétin-verse) + erreur

Si la condition d'égalité de variances n'est pas remplie, nous employons la transformation de Box et Cox (Box et al, 1978) : s'il est possible d'établir, entre la moyenne de la variable à expliquer et la variance associée pour chaque traitement expérimental, une relation de la forme $\ln ($ variance $)=a+b \ln ($ moyenne), alors la transformation à réaliser sur la variable à expliquer est la suivante :

$$
\text { variable }^{\text {transformée }}=\text { variable }^{(1-b / 2)}(\text { Box et al, 1978) }
$$

Nous n'avons retenu, dans le modèle final, que les facteurs et/ou covariables dont les valeurs des probabilités associées aux statistiques de test des hypothèses nulles sont inférieures à $\alpha=5 \%$ (risque de première espèce de rejeter l'hypothèse nulle alors qu'elle est vraie). Pour $\alpha>5 \%$, on considère que l'apport du facteur ou de la covariable dans le modèle n'est pas significatif et ces termes ont été éliminés successivement en commençant par le moins significatif.

Pour calculer les probabilités associées au test d'hypothèse nulle des différents facteurs et covariables du modèle, nous avons utilisé des sommes de carrés ajustées à l'ensemble des termes du modèle. Ces sommes de carrés permettent d'évaluer le niveau de signification d'un terme en présence de tous les autres et ne dépendent pas de l'ordre des termes dans le modèle.

Les effets des facteurs et covariables du modèle final sont alors estimés par résolution du système d'équations linéaires liées au modèle. Pour chaque modalité il est indiqué la variation moyenne de la variable de sortie due à cette modalité. Pour les covariables, la valeur du paramètre associé est déterminée.

Le logiciel de traitement statistique utilisé est SAS (Statistical Analysis System, SAS Institute Inc, 1989). La procédure utilisée pour les modèles linéaires est GLM (General Linear Model).

\section{COMPARAISON DES SUCCESSIONS CULTURALES}

\section{Classement des cultures en fonction de leur comportement vis-à-vis des pathogènes}

\section{Piétin-échaudage}

La comparaison des moyennes de gravité de maladie (tableau IV) permet surtout de distinguer 2 groupes de successions :

- celles ayant des céréales à paille (hiver ou printemps) parmi précédent ou antéprécédent (risque élevé) ; 
Tableau IV. Classement des successions culturales en fonction de la gravité des attaques de piétin-échaudage (moyenne de 25 plantes par parcelle).

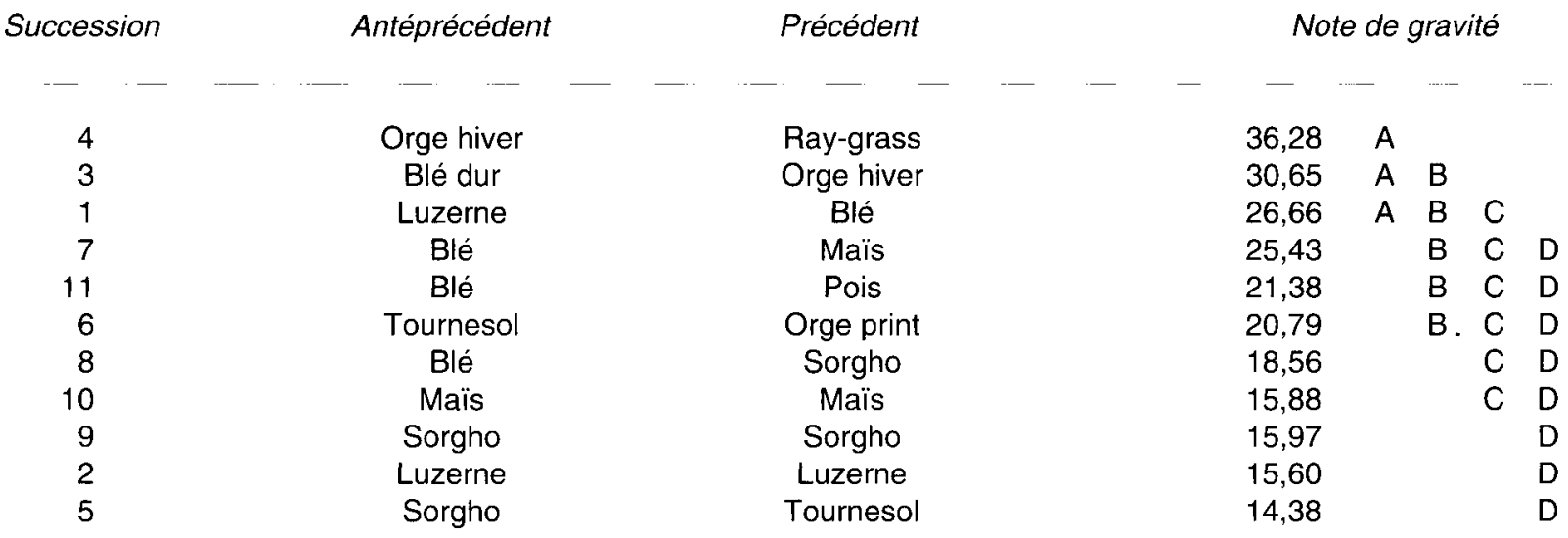

Les chiffres suivis d'une même lettre ne sont pas significativement différents (test de la plus petite différence significative ; $\alpha=0,05$ ). $r^{2}=0,35-F=35,27-P(H=0)=0,0001$.

- celles n'en ayant pas (successions $2,5,9$ et 10) (risque faible).

Une exception est à signaler : la succession 7 (blé/maïs) a un niveau d'infection supérieur à la succession 8 ayant cette même alternance de blé et culture non-hôte (blé/sorgho), alors que les successions 9 (sorgho/sorgho) et 10 (maïs/maïs) ne montrent pas de différence.

D'autre part, le ray-grass semble avoir un comportement différent des autres «non-céréales à paille» (différence significative entre successions 4 et 8 ayant toutes 2 un antéprécédent céréale d'hiver) et se rapprochant de celui des céréales à paille (différence non significative, entre successions 4 et 3 ayant toutes 2 un antéprécédent céréale d'hiver et comme précédent respectivement un ray-grass et une orge d'hiver).

\section{Piétin-verse}

La comparaison de moyennes 2 à 2 des pourcentages de plantes malades (tableau V) permet de distinguer essentiellement 3 groupes de successions culturales suivant la nature des précédents : - les successions à précédent céréales d'hiver (successions 1 et 3) (risque élevé) ;

Tableau V. Classement des successions culturales en fonction de la fréquence de plantes attaquées par le piétinverse (moyenne de 25 plantes par parcelle).

\section{Succession}

Antéprécédent

Luzerne
Blé dur
Tournesol
Blé
Orge hiver
Luzerne
Blé
Maïs
Blé
Sorgho
Sorgho

Précédent

Blé

Orge hiver

Orge print

Maïs

Ray-grass

Luzerne

Sorgho

Maïs

Pois

Tournesol

Sorgho
$\%$ plantes à piétin-verse

$\begin{array}{llll}51,4 & \text { A } & & \\ 48,6 & \text { A } & & \\ 27,5 & \text { B } & & \\ 26 & \text { B } & \text { C } & \\ 25,7 & \text { B } & \text { C } & \\ 11,4 & & \text { C } & \text { D } \\ 10,3 & & \text { C } & \text { D } \\ 8 & & & \text { D } \\ 7,49 & & & \text { D } \\ 4 & & & \text { D } \\ 1,7 & & & \text { D }\end{array}$

Les chiffres suivis d'une même lettre ne sont pas significativement différents (test de la plus petite différence significative, $\alpha=0,05$ ). $r^{2}=0,58-F=19,65-P(H=0)=0,0001$. 
- celles à précédent orge de printemps ou raygrass (successions 6 et 4 ) ;

- les autres (risque faible).

Cependant, une succession constitue une exception à ce classement : la succession 7 (blé/maiis) a un risque beaucoup plus élevé comparé à d'autres successions (successions 8 et 11) ayant cette même alternance de blé et de culture non-hôte (blé/sorgho ou blé/pois). En revanche, la succession 10 (maïs/maïs) se comporte de façon identique aux successions 9 (sorgho/sorgho) et 5 (sorgho/tournesol).

\section{Rhizoctone}

Il est difficile de séparer les successions (tableau $\mathrm{VI}$ ) aussi clairement que dans le cas du piétinverse ou du piétin-échaudage. Malgré cela, les successions culturales comprenant des céréales à paille (hiver ou printemps) se retrouvent généralement avec des niveaux d'infection plus élevés que les successions sans céréales. Cependant, la succession 3, comprenant 2 céréales d'hiver en précédent et antéprécédent, présente un niveau relativement faible de maladie. De plus, alors que les successions sans céréales en précédent et antéprécédent entraînent des niveaux très bas de maladie, la succession 5 à précédent tournesol et antéprécédent sorgho, se classe dans le groupe des successions avec céréales à paille.

D'autre part, le ray-grass ne semble pas avoir le même comportement qu'une céréale à paille (différence entre successions 4 et 3 où 2 céréales d'hiver précèdent le ray-grass et l'orge d'hiver).

\section{Classement des cultures en fonction de leur influence sur les maladies}

Les essais montrent que toutes les céréales à paille augmentent le risque de piétin-échaudage et de piétin-verse. Pour ce dernier, les céréales d'hiver semblent engendrer un risque plus élevé que les céréales de printemps et le ray-grass se comporte comme une céréale de printemps. En revanche, dans le cas du piétin-échaudage, le ray-grass diffère des céréales à paille (succession céréale à paille/ray-grass engendrant un risque même supérieur à la succession céréale à paille/céréale à paille) et encore plus des «noncéréales à paille".

Le maïs a également un comportement différent des autres "non-céréales à paille», il augmente le risque de piétin-échaudage et de piétinverse (augmentation du risque en cas de succession blé/maiis) sans en être porteur (pas d'augmentation du risque en cas de succession maïs/maïs).

Sa situation à part ne s'explique pas par le labour : s'il y a eu possibilité d'une remontée de vieilles pailles provenant d'un antéprécédent blé, la succession blé/sorgho l'a également permis sans indice élevé de maladie. Elle ne peut pas non plus être due à la fertilisation azotée élevée,

Tableau VI. Classement des successions culturales en fonction de la fréquence de plantes attaquées par le rhizoctone (moyenne de 25 plantes par parcelle).

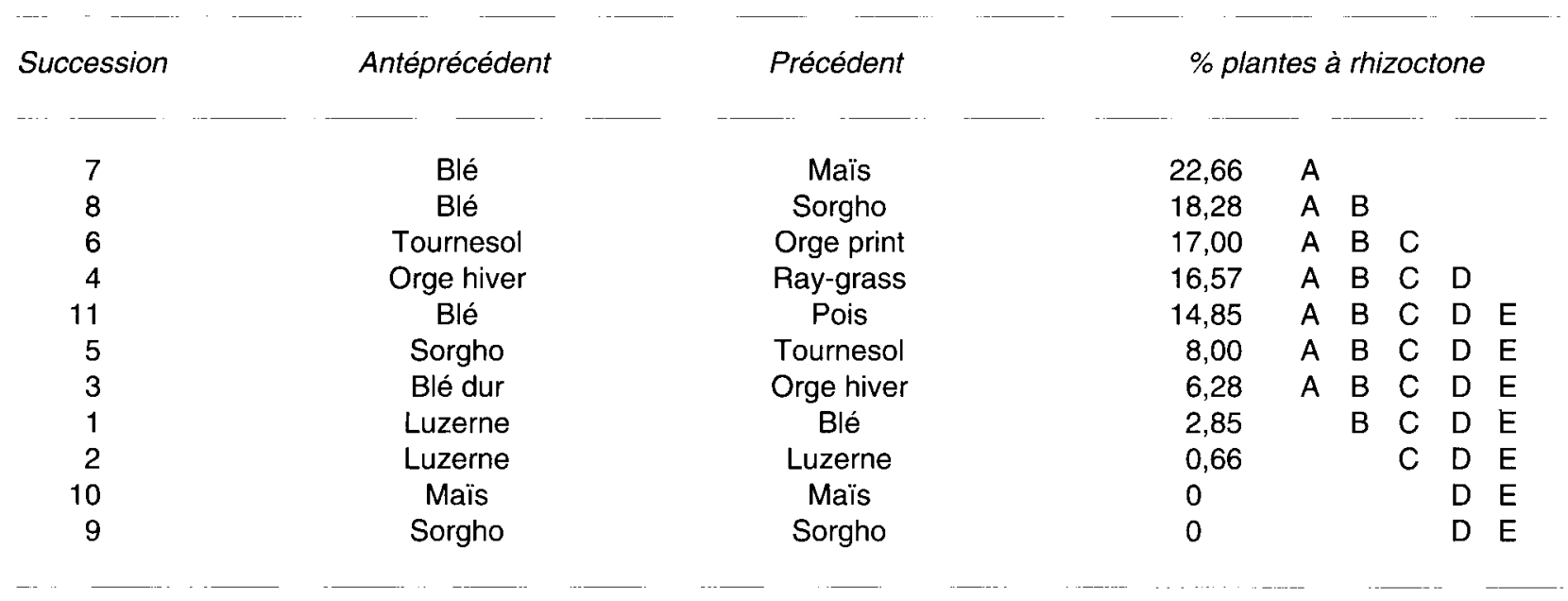

Les chiffres suivis d'une même lettre ne sont pas significativement différents (test de la plus petite différence significative ; $\alpha=0,05$ ). $r^{2}=0,23-F=5,49-P(H=0)=0,0001$. 
puisque non seulement tous les blés disposaient d'une quantité totale d'azote identique, mais en plus la quantité d'azote absorbée par le blé derrière maïs (174 $\pm 12 \mathrm{~kg} / \mathrm{ha}$ ) n'est pas significativement différente de celle derrière sorgho (165 \pm $12 \mathrm{~kg} / \mathrm{ha}$ ) au risque $\alpha=5 \%$.

Pour rendre compte de cette différence de comportement du maïs, nous avons créé une nouvelle classe de cultures qu'on appellera les cultures «amplificatrices». Cette classe regroupera toutes les cultures amplifiant un risque de maladies dû à d'autres cultures présentes dans la succession, sans être nécessairement des cultures hôtes. Cette classe est restreinte au maïs pour le piétin-verse, mais comprend également le ray-grass dans le cas du piétin-échaudage. Les autres cultures (tournesol, luzerne, pois, sorgho) sont des cultures non-hôtes sans influence particulière.

L'augmentation de maladie en cas de présence de céréales à paille n'est pas aussi claire pour le rhizoctone que pour le piétin-verse ou le piétinéchaudage. II est possible que cette absence de tendances nettes soit due à une interaction du rhizoctone avec le piétin-verse.

L'analyse des successions montre que maïs, sorgho et ray-grass ont des comportements particuliers. Cependant, pour les 2 premiers, il peut aussi s'agir d'une influence du travail du sol puisque ce sont les 2 seuls précédents suivis d'un labour avant l'installation du blé noté. Cette particularité pourrait être responsable de l'augmentation du risque de rhizoctone : il s'agirait alors d'un effet travail du sol et non d'un effet nature du précédent. Mais nos essais ne permettent pas de distinguer entre ces 2 effets. Sur la base de nos résultats et par analogie avec les 2 autres maladies, ray-grass, maïs et sorgho constitueront le groupe des cultures "amplificatrices", tandis que toutes les autres non-céréales à paille formeront le groupe des non-hôtes.

Les précédents et antéprécédents ont ainsi été classés en plusieurs groupes suivant leur influence sur les maladies, classement qui est résumé par le tableau VII.

\section{MODÉLISATION DE L'EFFET DES SUCCESSIONS CULTURALES SUR L'EXPRESSION DES MALADIES}

La répartition des cultures, suivant leur influence sur les maladies, trouve son expression dans des modèles linéaires expliquant le niveau de maladie en fonction du précédent et de l'antéprécédent, hiérarchisé sous l'effet du précédent, de l'irrigation et d'un certain nombre de covariables. La nécessité de hiérarchiser l'antéprécédent sous l'effet précédent est illustrée par le tableau VIII qui reflète le déséquilibre du dispositif expérimental.

Afin de vérifier la validité de la classe «amplificatrice», nous avons appliqué ce même modèle en utilisant la répartition classique : hôtes opposés à non-hôtes, les premiers regroupant les céréales à paille (et le ray-grass dans le cas du piétin-verse), les deuxièmes les autres cultures ; ou en adoptant la répartition en 3 groupes: hôtes, amplificatrices, non-hôtes.

Tableau VII. Classement des cultures en fonction de leur influence sur l'occurrence des maladies du pied et des racines du blé.

Groupe

Hôte

Culture amplificatrice

Non-hôte
Piétin-échaudage

Blé et orge d'hiver

Orge de printemps

Maïs

Ray-grass

Tournesol

Luzerne

Pois

Sorgho
Rhizoctone

Blé et orge d'hiver

Orge de printemps

Maïs

Ray-grass

Sorgho

Tournesol

Luzerne

Pois

\section{Piétin-verse}

Fort: Blé et orge d'hiver

Faible: Orge de printemps Ray-grass

Maïs

Tournesol

Luzerne

Pois

Sorgho 
Tableau VIII. Tableau d'effectifs des combinaisons précédent*antéprécédent pour les 3 maladies étudiées.

\section{A. Piétin-échaudage}

Précédent $\quad \rightarrow \quad$ Hôte Amplificateur Non-hôte

Antéprécédent

Hôte
Amplificateur
Non-hôte

6

15

21

\section{B. Piétin-verse}

\section{Précédent} $\rightarrow$

Hôte fort

\section{Non-hôte}

Antéprécédent

Hôte fort
Amplificateur
Non-hôte

Total

\section{Rhizoctone}

Hôte

Amplificateur

$\begin{array}{rr}\text { Total } & \\ 14 & 33 \\ & 6 \\ 20 & 35 \\ 34 & 74\end{array}$

$\rightarrow$

Précédent

7

13

Antéprécédent

Hôte

Amplificateur

Non-hôte
6

15

21
20

13

33
6

6

8

15

12

\section{Total}

14

20

34
33

6

35

74
Malheureusement l'anté-antéprécédent n'est pas représenté dans ces modèles, puisque la structure des successions n'est pas assez diversifiée pour permettre son étude. D'autre part, la durée de survie saprophytique des champignons, qui est d'un an pour le piétin-échaudage (Steinbrenner et Höflich, 1980) et d'environ 2 à 3 ans pour le piétin-verse (Macer, 1961 ; Steinbrenner et Höflich, 1980), permet de considérer l'influence de l'anté-antéprécédent comme faible ou même négligeable. En revanche, la durée de survie saprophytique du rhizoctone n'est pas connue.

\section{Piétin-échaudage}

Quelle que soit la répartition de cultures utilisée, le modèle retenu est le suivant :

piétin-échaudage (\% système racinaire 'nécrosé moyen $)=$ irrigation + précédent + précédent *antéprécédent

Le modèle utilisant la répartition avec cultures amplificatrices expliquant le plus de variabilité $\left(\mathrm{r}^{2}\right.$ $=0,36$ contre $r^{2}=0,26$ ), nous n'exposerons ici que celui-ci. Afin de faciliter l'analyse des résul- 
Tableau IX. Modèle linéaire retenu pour expliquer la gravité d'infection par le piétin-échaudage.

A. Tableau d'analyse de variance

\begin{tabular}{|c|c|c|c|c|c|c|}
\hline Source & Degré de liberté & Somme de carrés & Carré moyen & Valeur de $F$ & $\operatorname{Pr}(F>F o b s)$ & $r^{2}$ \\
\hline Modèle & 6 & 0,3406 & 0,0567 & 6,16 & 0,0001 & 0,36 \\
\hline Erreur & 66 & 0,6070 & 0,0092 & & & \\
\hline Total corrigé & 72 & 0,9477 & & & & \\
\hline \multicolumn{7}{|l|}{ Effets } \\
\hline Irrigation & 1 & 0,0635 & 0,0635 & 6,91 & 0,0107 & \\
\hline Précédent & 2 & 0,129 & 0,0649 & 7,06 & 0,0017 & \\
\hline Antéprécédent ${ }^{\star}$ précédent & 3 & 0,155 & 0,0516 & 5,61 & 0,0017 & \\
\hline
\end{tabular}

B. Estimateurs des effets présents dans le modèle final

Constante

Irrigation

Précédent

Antéprécédent

Hôte

Amplificatrice

Non-hôte
13,36

$\begin{array}{cc}\begin{array}{c}\text { Présence } \\ 6,94\end{array} & \text { Absence } \\ & 0 \\ \text { Hôte } & \text { Amplificatrice }\end{array}$

16,37

0,29
Non-hôte

4,22

0

Piétin-échaudage $=$ constante + effet irrigation + effet précédent + effet antéprécédent hiérarchisé sous l'effet précédent. Les estimateurs en gras sont les seuls qui sont considérés comme significativement différents de 0 au risque $\alpha=0,05$ associé au test de l'hypothèse nulle.

tats, nous avons choisi de présenter les résultats

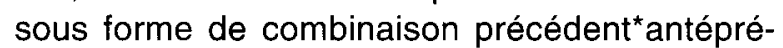
cédent, sans faire ressortir l'effet principal du précédent.

Aucune covariable n'est significative au niveau $\alpha=5 \%$ (tableau IX) ; seuls les effets précédent, antéprécédent et irrigation le sont. La combinaison antéprécédent*précédent a d'ailleurs le même niveau de signification que l'effet précédent (probabilité associée au test d'hypothèse nulle égale à $0,0017 \%$ ), ce dernier n'expliquant que $28 \%$ de la variabilité $\left(r^{2}=0,28\right.$ pour un modèle $g p e=$ précédent + irrigation $)$.

\section{Piétin-verse}

II a été nécessaire d'employer une transformation de Box et Cox. Quelle que soit la répartition des cultures utilisées, le modèle retenu est le suivant : piétin-verse ( $\%$ plantes malades)transformé $=$ précédent + précédent*antéprécédent

Cependant, le dernier terme n'est significatif qu'à $10 \%$, quelle que soit la répartition des cultures utilisée. Nous avons cependant choisi de le garder dans le modèle puisque, sans sa présence, la qualité du modèle diminuerait de façon non négligeable. Les covariables et le facteur irrigation ne sont pas significatifs, ni au seuil de $5 \%$, ni au seuil de $10 \%$.

Nous n'exposons ici que le modèle différenciant les cultures amplificatrices dont la qualité est également la meilleure $\left(r^{2}=0,52\right.$ contre $r^{2}=$ 0,48 ). Les résultats sont présentés sous forme de combinaison précédent ${ }^{\star}$ antéprécédent (tableau $X)$. Le poids du précédent est plus important que pour le piétin-échaudage : le modèle réduit au seul terme piétin-verse = précédent explique $46 \%$ de la variabilité $\left(r^{2}=0,46\right)$ contre $52 \%$ expliqué 
Tableau X. Modèle linéaire retenu pour expliquer le pourcentage de plantes à symptômes du piétin-verse.

A. Tableau d'analyse de variance

\begin{tabular}{lcccccc}
\hline Source & Degré de liberté & Somme de carrés & Carré moyen & Valeur de $F$ & $\operatorname{Pr}(F>F o b s)$ & $r^{2}$ \\
\hline $\begin{array}{l}\text { Modèle } \\
\text { Erreur }\end{array}$ & 7 & 2,92 & 0,417 & 10,30 & 0,0001 & 0,52 \\
Total corrigé & 66 & 2,67 & 0,0405 & & & \\
& 73 & 5,58 & & & \\
Effets & & & & & \\
$\quad$ Précédent & 3 & 2,41 & 0,803 & 19,83 & 0,0001 \\
$\quad$ Antéprécédent*précédent & 4 & 0,332 & 0,083 & 2,05 & 0,0967 \\
& & & & & \\
\hline
\end{tabular}

B. Estimateurs des effets présents dans le modèle final pour la variable piétin-verse(1-1,221/2)

\section{Constante}

Précédent
Antéprécédent
Hôte
Amplificatrice
Non-hôte

Hôte $\quad$ Hôte
fort
faible

3,06

3,34

faible

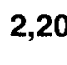

2,11

\section{1,23}

Amplificatrice

Non-hôte
2,05

0,44
0,70

0

Piétin-verse ${ }^{(1-1,221 / 2)}=$ constante + effet précédent + effet antéprécédent hiérarchisé sous l'effet précédent (transformation de Box et Cox (Box et $a l, 1978$ ) nécessaire puisque la relation In(variance) $=a+b \ln$ (moyenne) est significative avec $b=1,221$ ). Les estimateurs en gras sont les seuls qui sont considérés comme significativement différents de 0 au risque $\alpha=0,05$ associé au test de l'hypothèse nulle.

par le modèle retenu piétin-verse $=$ précédent + précédent ${ }^{\star}$ antéprécédent $\left(r^{2}=0,52\right)$ pour lequel, en plus, le dernier terme n'est significatif qu'à $10 \%$.

\section{Rhizoctone}

Il a également été nécessaire d'utiliser une transformation de Box et Cox. Comme pour le piétinéchaudage et le piétin-verse, le modèle intégrant la classe des amplificatrices est le meilleur $\left(r^{2}=\right.$ 0,36 contre $\left.r^{2}=0,24\right)$ et nous nous limiterons à son étude. Le modèle retenu est le suivant :

rhizoctone (\% plantes malades) $)^{\text {transformé }}=a^{*}$ piétin-verse (\% plantes malades) + précédent + précédent*antéprécédent

Comme dans le cas du piétin-échaudage, nous avons privilégié l'aspect combinaison précédent*antéprécédent par rapport à l'effet principal précédent lors de la présentation des résultats (tableau XI).
$\mathrm{Ni}$ les covariables relatives au peuplement (plantes par $\mathrm{m}^{2}$, azote absorbé) ni l'effet irrigation ne sont significatifs. La combinaison antéprécédent*précédent a d'ailleurs le même niveau de signification que l'effet précédent (probabilité associée au test d'hypothèse nulle égale à $0,01 \%)$. Le modèle réduit au simple précédent rhizoctone $=$ précédent $+a^{*}$ piétin-verse n'est pas lui non plus significatif.

Le terme $\mathrm{a}^{*} p v(\%)$ est rajouté pour rendre compte de la corrélation négative entre piétinverse et rhizoctone. II n'y a, en revanche, pas eu de terme $\mathrm{a}^{*} r(\%)$ dans le modèle du piétin-verse, puisque le piétin-verse est considéré comme étant le champignon dominant.

\section{DISCUSSION ET CONCLUSION}

Malgré la diversité des précédents, la conduite uniforme du blé suivant, à l'exception près de l'irri- 
Tableau XI. Modèle linéaire retenu pour expliquer le pourcentage de plantes à symptômes de rhizoctone.

A. Tableau d'analyse de variance

\begin{tabular}{|c|c|c|c|c|c|c|}
\hline Source & é de liberté & Somme de carrés & Carré moyen & Valeur de $F$ & $\operatorname{Pr}($ F $>$ Fobs $)$ & $r^{2}$ \\
\hline Modèle & 7 & 33,3 & 4,76 & 5,23 & 0,0001 & 0,36 \\
\hline Erreur & 66 & 60,1 & 0,91 & & & \\
\hline Total corrigé & 73 & 93,4 & & & & \\
\hline \multicolumn{7}{|l|}{ Effets } \\
\hline Précédent & 2 & 7,59 & 3,8 & 4,17 & 0,0195 & \\
\hline Antéprécédent ${ }^{*}$ précédent & 4 & 28,59 & 7,15 & 7,85 & 0,0001 & \\
\hline Piétin-verse & 1 & 3,64 & 3,64 & 4,00 & 0,0496 & \\
\hline
\end{tabular}

B. Estimateurs des effets présents dans le modèle final pour la variable rhizoctone ${ }^{(1-1,415 / 2)}$

\begin{tabular}{|c|c|c|c|}
\hline Constance & 0,89 & & \\
\hline Piétin-verse & $-0,01425$ & & \\
\hline Précédent & Hôte & Amplificatrice & Non-hôte \\
\hline \multicolumn{4}{|l|}{ Antéprécédent } \\
\hline Hôte & 1,30 & 1,38 & 0,39 \\
\hline Amplificatrice & & $-0,59$ & 0,31 \\
\hline Non-hôte & 1,40 & & 0 \\
\hline
\end{tabular}

gation, a permis l'atteinte d'états du peuplement suffisamment proches pour que ceux-ci n'interviennent pas de façon significative dans le déroulement des maladies (absence des covariables de peuplement dans les modèles finaux).

Les périodes humides favorisent le développement du piétin-échaudage et du piétin-verse. Elles permettent une humidité suffisante du sol pour le développement du premier (Garrett, 1934 ; Cook et al, 1972 ; Asher et Shipton, 1981) et favorisent la sporulation et l'infection du deuxième (Ponchet, 1959 ; Schrödter et Fehrmann, 1971a, b). Cependant, il n'a pas été observé d'effet de l'irrigation dans le cas du piétin-verse. II est probable que l'irrigation réalisée en période chaude et sèche n'a pas permis d'obtenir les conditions nécessaires à la sporulation, à savoir $15 \mathrm{~h}$ à $85 \%$ d'humidité relative et une température comprise entre 4 et $13^{\circ} \mathrm{C}$
(Fehrmann, Schrödter, 1971a, b ; Rapilly et al, 1979). Il est également possible que l'irrigation intervienne trop tard dans le cycle pour que les infections par $P$ herpotrichoides résultant d'une éventuelle sporulation puissent encore se traduire par des nécroses. Il n'en est pas de même pour le piétin-échaudage, l'infection par le mycélium pouvant être réalisée à tout moment du cycle et évoluer rapidement en symptômes. Pour cette maladie, l'effet de l'irrigation est significatif. Ainsi, l'effet des irrigations pourrait dépendre fortement des dates auxquelles elles sont réalisées, variables en fonction de l'année de culture.

Il n'y a, en revanche, par d'effet irrigation pour le rhizoctone. Ce champignon est surtout abondant en années sèches (Reinecke et Fehrmann, 1979), car il se développe mieux dans les sols secs (Pitt, 1964 ; Hanrion et Cabanettes, 1983) même si l'humidité lui est indispensable après 
l'installation sur la plante (Cavelier et al, 1982). Le rhizoctone est donc moins dépendant de l'humidité que les 2 autres pathogènes.

L'effet dominant du piétin-verse sur le rhizoctone ayant été pris en compte par le terme $a^{*} p v$, l'interaction entre ces 2 champignons, avec dominance du premier sur le dernier, se traduit alors par une corrélation négative qui a également été observée par d'autres auteurs (Reinecke et Fehrmann, 1979 ; Van der Hoeven et Bollen, 1980 ; Cavelier et al, 1985). Les 2 agents pathogènes infectent le même site sur la plante et il y a compétition pour les mêmes tissus (Reinecke et al, 1979) et possibilité d'un antagonisme, même si celui-ci a été uniquement observé in vitro (Brück et Schlösser, 1982). Cependant, ces derniers auteurs indiquent que les 2 parasites peuvent cohabiter sur une même lésion.

Malgré ces quelques différences, les structures des modèles des 3 maladies sont comparables. L'effet antéprécédent n'apparaît que faiblement significatif dans le cas du piétin-verse alors qu'il est hautement significatif dans le cas du piétin-échaudage et du rhizoctone. II est intéressant d'observer que des 3 maladies, ces 2 dernières sont les seules dont le développement ne passe pas par la production et la dissémination de spores. L'effet antéprécédent semble donc important pour des maladies à évolution lente pour lesquelles la multiplication de l'inoculum sur plusieurs cultures hôtes successives est nécessaire pour obtenir un niveau d'attaque important.

Il est également possible que le climat peu favorable du site d'essai n'ait pas permis une infection importante de l'antéprécédent hôte par le piétin-verse. La quantité d'inoculum laissée et donc l'effet sur le blé noté sont par conséquence très faibles.

De même, le classement des successions varie en fonction des maladies. Pour le piétinéchaudage, c'est-à-dire la maladie pour laquelle dissémination et infection se font entièrement dans le sol, les successions comprenant au moins une culture hôte et pas de culture non-hôte parmi précédent et antéprécédent ont un niveau de maladie très important. On remarque que les combinaisons hôte/amplificatrice ont un niveau comparable à celui des combinaisons hôte/hôte alors qu'on pourrait s'attendre à un risque plus élevé dans le dernier cas à cause d'une quantité d'inoculum plus importante. Mais l'effet amplificateur du maïs et du ray-grass compense largement cette différence de quantité d'inoculum initial.
Les successions comportant une culture hôte et une culture non-hôte, parmi précédent et antéprécédent, et donc pas de culture "amplificatrice" ont un niveau de piétin-échaudage plus faible. En cas de précédent hôte et antéprécédent non-hôte, le risque est beaucoup plus important qu'en cas inverse, c'est-à-dire précédent non-hôte et antéprécédent hôte. Le champignon est en effet très peu compétitif lors de sa phase de survie saprophytique et une année de culture non-hôte réduit de façon sensible l'inoculum du sol (Steinbrenner et Höflich, 1980 ; Shipton, 1981).

Pour le rhizoctone dont la dissémination et l'infection ne se font que partiellement dans le sol, c'est l'effet précédent qui influence le plus le classement des successions. Après corrélation des résultats de l'effet piétin-verse, les successions à précédent hôte s'avèrent être celles à risque maximal. Cependant, les successions à précédent amplificateur et antéprécédent hôte ont un niveau de maladie comparable aux successions à précédent hôte, l'effet amplificateur compensant la différence de quantité initiale d'inoculum. En revanche, les successions à précédent nonhôte et antéprécédent hôte ont un niveau de maladie beaucoup plus bas, laissant penser qu'une culture non-hôte et non amplificatrice réduit significativement la quantité d'inoculum capable d'infecter.

Dans le cas du piétin-verse, c'est-à-dire la maladie pour laquelle dissémination et infection sont presque entièrement localisées en-dehors du sol, les successions ayant un précédent hôte sont celles à risque majeur, avec un risque encore plus élevé en cas de précédent hôte fort. Dans ce cas, l'antéprécédent est alors sans influence. La quantité d'inoculum laissée par le précédent est alors tellement importante que celle provenant de l'antéprécédent aura un effet négligeable.

L'antéprécédent ne semble avoir un effet que si le précédent n'est pas un hôte. Dans ce cas-là, les seuls antéprécédents ayant une influence paraissent être les hôtes forts. Si en plus le précédent est une culture amplificatrice, le risque de maladie est augmenté à tel point qu'on obtient un niveau comparable à celui des successions à précédent hôte faible. En cas de précédent non-hôte, il n'y a pas d'effet amplificateur et le niveau de maladie est plus faible. On ne connaît pas l'influence des hôtes faibles qui n'étaient pas présents en tant qu'antéprécédent dans les successions étudiées.

Malgré ces différences, ce sont toujours les mêmes types de succession qui présentent le 
risque le plus faible, quelle que soit la maladie : il s'agit des successions ne comprenant aucune culture hôte parmi précédent et antéprécédent. Les successions à précédent non-hôte ont toujours un niveau d'infection faible.

Le modèle présenté pour le piétin-échaudage n'est valable que pour les successions mixtes comprenant céréales à paille et "non-céréales à paille", car en cas de monoculture de céréales à paille, non abordée dans notre étude, le phénomène du déclin (Shipton, 1972) se manifeste : le pic de maladie se situe au niveau de la troisième ou quatrième culture consécutive, au-delà de laquelle il y a diminution de l'infection jusqu'à un niveau affectant peu le rendement.

Les modèles présentés pour le piétin-échaudage et le piétin-verse sont conformes aux classements observés par Steinbrenner et Obenauf (1986) et Steinbrenner et al (1990) pour le piétinéchaudage ; par Steinbrenner (1981) ainsi que Groll et Luzi (1991) pour le piétin-verse. Ces derniers ne distinguent cependant pas les 2 catégories d'hôtes en fonction de leur sensibilité au piétin-verse, ni les 2 groupes de non-hôtes (amplificateurs ou non). II se confirme ici que précédent et antéprécédent doivent être considérés conjointement, à cause de l'interaction constante entre quantité d'inoculum disponible et autres mécanismes, tels qu'importance de la microflore antagoniste, surtout dans le cas de champignons à dissémination et infection à l'intérieur du sol. Une même quantité d'inoculum peut être à l'origine de niveaux de maladies très variables en fonction de l'importance de la microflore antagoniste : si cette dernière est très développée, la maladie s'exprime peu, même en cas de quantité d'inoculum élevée. Inversement, si la microflore est peu développée, une faible quantité d'inoculum peut déjà être à l'origine d'une explosion de maladie.

Malgré ces différences dans le classement des successions, les cultures hôtes sont plus ou moins identiques pour les 3 agents pathogènes: les céréales à paille en font toujours partie, ce qui est confirmé par la littérature (Glynne, 1950 ; Pitt, 1964 ; Steinbrenner et al, 1990). Le piétinverse attaque cependant moins les céréales de printemps (Steinbrenner et al, 1990), probablement parce qu'en cas de céréale de printemps les conditions climatiques sont moins favorables à la sporulation du champignon, puis à la contamination des plantes. D'où le classement de l'orge de printemps parmi les hôtes faibles dans le cas du piétin-verse. Nous y avons mis également le ray-grass qui, bien que "non-céréale à paille», est également une culture hôte du piétin-verse (Ponchet, 1959 ; Wehner et al, 1988).

Le comportement du ray-grass vis-à-vis du piétin-échaudage est ambigu. II peut être une culture hôte (Garrett, 1956 ; Slope et Etheridge, 1971) ou stimuler la microflore antagoniste du piétin-échaudage (Wehrle et Ogilvie, 1955 ; Scott, 1970), comme par exemple Phialophora radicicola (Scott, 1970 ; Deacon, 1973 ; Slope et al, 1979 ; Clarkson et Polley, 1981). Il s'agit d'une culture dont le rôle amplificateur ne ressort qu'en cas de présence de forte pression d'inoculum, c'est-à-dire en association avec des cultures hôtes. En leur absence, le ray-grass se comporte comme un non-hôte. II n'y a pas de renseignement concernant le rhizoctone, mais il est possible que dans ce cas le comportement du raygrass soit analogue à celui observé avec le piétin-échaudage.

À cause de son comportement différent aussi bien de celui des céréales à paille que de celui des autres "non-céréales à paille», le maïs est toujours considéré comme une culture «amplificatrice", quel que soit le champignon étudié. Dans le cas du piétin-échaudage, le maïs est responsable d'une augmentation de la réceptivité du sol à la maladie (Lucas et al, 1989). La notion de réceptivité est définie par Alabouvette et al (1982) comme étant l'aptitude d'un sol à permettre l'expression des capacités pathogènes d'un inoculum sur une population de plantes hôtes sensibles. Lorsqu'il y a un apport d'inoculum de $G$ graminis, ce qui est le cas lors d'une combinaison hôte*maïs, il y a amplification de l'infestation.

Dans le cas du piétin-verse, les raisons du comportement du maïs restent inconnues. Pour le rhizoctone, la littérature ne fournit pas non plus d'indication, mais il est possible qu'il s'agisse d'un mécanisme comparable à celui indiqué pour le piétin-échaudage.

Le phénomène des cultures «amplificatrices", observé jusqu'à présent uniquement dans le cas du piétin-échaudage, a pu être mieux cerné. II s'agit de cultures dont l'effet favorable sur les maladies ne s'exprime qu'en association avec des cultures hôtes. Cette notion qui rend compte d'effets autres que la quantité d'inoculum laissée par la succession culturale, a pu être élargie au piétin-verse et au rhizoctone, même si, dans ces cas, les mécanismes restent à identifier afin de s'assurer de la stabilité des phénomènes observés.

II reste cependant quelques lacunes dans ces modèles. 
Les conditions climatiques de Toulouse n'étant pas parmi les plus favorables pour les maladies étudiées, il est possible que certaines différences ne se soient pas exprimées d'une manière suffisamment importante pour être significatives du point de vue statistique. Dans des situations plus favorables au piétin-verse, il est, par exemple, possible que l'antéprécédent agisse lui-aussi de façon significative sur la maladie.

Le nombre de combinaisons de cultures n'a pas été suffisant pour étudier tous les effets. Dans le cas du piétin-verse, on ne peut en effet conclure sur l'influence d'un hôte faible en position d'antéprécédent, puisque cette situation ne s'est pas trouvée sur l'essai. D'autre part, on n'a pas pu expliquer les effets éventuels de l'antéantéprécédent, même si les durées de survie saprophytiques du piétin-verse et piétin-échaudage, inférieures à la durée de temps entre l'antéantéprécédent et le blé étudié, ne le justifient pas nécessairement. En revanche, la durée de survie du rhizoctone reste toujours inconnue.

Peu de non-céréales à paille ont été prises en compte (luzerne, tournesol, sorgho, maïs, pois). II semble par exemple que le colza augmente également le risque de piétin-verse par une stimulation de la sporulation (Lange-de la Camp et Naumann, 1978 ; Schulz et al, 1990). De même, au sein des céréales à paille, il existe une variabilité de sensibilité qu'il serait intéressant de prendre en compte, qu'elle soit intra- (variétés plus ou moins sensibles au piétin-verse) ou interespèce (avoine peu sensible aux piétin-verse et piétin-échaudage : Garrett, 1934 ; Slope et Etheridge, 1971 ; Steinbrenner et al, 1990).

Nos modèles ne prennent pas en compte les monocultures de céréales qui sont intéressantes surtout pour le piétin-échaudage à cause d'une diminution de la réceptivité du sol au champignon. II faudrait compléter les résultats de ce travail afin de pouvoir proposer des modèles complets pour le classement des successions culturales en fonction des risques pathologiques.

Les mécanismes responsables de l'effet amplificateur du maïs dans le cas du piétin-verse et du rhizoctone, ainsi que celui du sorgho dans le cas du rhizoctone, n'ont pas pu être expliqués. II est donc possible qu'il ne s'agisse pas d'un effet "stable" qui se reproduira ou pas dans d'autres conditions.

Nos résultats devront donc être complétés à l'aide d'essais mis en place sur des sites plus favorables aux maladies étudiées, combinant un nombre plus élevé de cultures, notamment de "non-céréales à paille», et permettant la prise en compte de l'effet anté-antéprécédent Parallèlement, le comportement des cultures dites «amplificatrices» devra être analysé plus en détail, en suivant notamment les états intermédiaires des parasites et autres microorganismes du sol pendant l'évolution de la maladie.

\section{REMERCIEMENTS}

Les auteurs remercient JR Marty et son équipe de I'INRA de Toulouse-Auzeville qui sont à l'origine de la mise en place de l'essai.

\section{RÉFÉRENCES}

Alabouvette C, Couteaudier Y, Louvet J (1982) Comparaison de la réceptivité de différents sols et substrats de culture aux fusarioses vasculaires. agronomie 2, 1-6

Asher MJC, Shipton PJ (1981) Biology and control of take-all. In : Biology and control of take-all (MJC Asher, PJ Shipton, eds), Academic Press, London, 317-341

Bodker L, Schulz H, Kristensen K (1990) Influence of cultural practices on incidence of take-all (Gaeumannomyces graminis var triticl) in winter wheat and winter rye. Tidsskr Planteav/ 94, 201-209

Box GEP, Hunter WG, Hunter JS (1978) Statistics for experimenters: an introduction to design, data analysis and model building. Wiley, New York, 653 $p$

Brück KP, Schlösser E (1982) Getreidefußkrankheitserreger. $V$. Antagonismus zwischen den Erregern. Z Pflanzenkr Pflanzenschutz 89, 337-343

Cavelier N, Lucas P, Perraton B (1982) Maladies du pied. Cultivar 15, 6-19

Cavelier N, Lucas P, Boulch G (1985) Évolution du complexe parasitaire constitué par Rhizoctonia cerealis et Pseudocercosporella herpotrichoides, champignons parasites de la base des tiges des céréales. agronomie 5, 693-700

Clarkson JDS, Polley RW (1981) Diagnosis, assessment, crop-loss appraisal and forecasting. In : Biology and control of take-all (MJC Asher, PJ Shipton, eds), Academic Press, London, 251-269

Cook RJ, Pappendick RI, Griffin DM (1972) Growth of 2 root-rot fungi as affected by osmotic and matric water potentials. Soil Sci Soc Am Proc 36, 78-82

Deacon JW (1973) Factors affecting occurrence of the Ophiobolus patch disease on turf and its control by Phialophora radiciola. Plant Pathol 22, 149-155

Fitt BDL, Lysandrou M (1984) Studies on mechanism of splash dispersal of spores, using Pseudocercosporella herpotrichoides spores. Phytopathol Z 111, 323-331 
Garrett SD (1934) Factors affecting the severity of take-all. III. The climatic factor. J Dep Agric S Aust 37, 976-983

Garrett SD (1956) Biology of root-infecting fungi. Cambridge University Press, Cambridge, UK, $294 \mathrm{p}$

Glynne MD (1950) Sharp eyespot as a severe disease of oats. Nature 166, 232-236

Groll U, Luzi K (1991) Untersuchungen zum Einfluß acker- und pflanzenbaulicher Faktoren auf den Halmbruchbefall an Wintergetreide. Arch Phytopathol Pflanzensch 27, 459-470

Hanrion M, Cabanettes JP (1983) Rhizoctone des céréales. Cultivar 167, 74

Huet P (1986) Résultats d'expérimentations systématiques sur les maladies fongiques d'origine tellurique. In: Les résistances génétiques des cultures céréalières, Versailles 23-24 janvier 1986, INRA Paris. Les colloques de l'INRA 35, 163-177

Kapoor IJ, Hoffmann GM (1984) Antagonistic action of Pseudocercosporella herpotrichoides on Ceratobasidium $\mathrm{sp}$ and Rhizoctonia solani associated with foot rot of cereals. $Z$ Pflanzenkr Pflanzenschutz 91, 250-257

Lange-de la Camp M, Naumann K (1978) Wirkung von Pflanzenrückständen auf die parasitische Aktivität von bodenbürtigen Krankheitserregern und die saprophytische Mikroflora des Bodens, III. Einfluß von Roggen und Raps als Winterzwischenfrüchte auf den Befall mit Cercosporella herpotrichoides Fron. Zbl Bakt /l Abt 133, 286-312

Lucas P, Sarniguet A, Collet JM, Lucas M (1989) Réceptivité du sol au piétin-échaudage (Gaeumannomyces graminis var triticl) : influence de certaines techniques culturales. Soil Biol Biochem 21(8), 1073-1078

Macer RCF (1961) Survival of Cercosporella herpotrichoides Fron in wheat straw. Ann Appl Biol 49, 165172

Pitt D (1964) Studies on sharp eyespot disease of cereals. I. Disease symptoms and pathogenicity of isolates of Rhizoctonia solani Kühn and the influence of soil factors and temperature on disease development. Ann App/ Biol 54, 77-89

Polley RW, Thomas MR (1991) Surveys of diseases of winter wheat in England and Wales, 1976-1988. Ann Appl Biol 119, 1-20

Ponchet J (1959) La maladie du piétin-verse des céréales, importance agronomique, biologie et épiphytologie. Ann Epiphyt 45-98

Prew RD (1977) Studies on the spread, survival and control of take-all and other foot and root diseases of wheat and barley. Ph D Thesis, University of London, UK

Prew RD (1981) Cropping system in relation to soilborne and trash-borne diseases of cereals. In Strategies for the control of cereal diseases (JF Jenkin, RT Plumb, eds), Blackwell, Oxford, UK, 149-156
Reinecke H, Fehrmann H (1979) Rhizoctonia cerealis Van der Hoeven an Getreide in der BRD. Z Pflanzenkr Pflanzenschutz 86, 190-204

Reinecke P, Duben J, Fehrmann J (1979) Antagonism between fungi of the foot rot complex of cereals. In : Soilborne plant pathogens (B Schippers, W Gams, eds), Academic Press, New York, États-Unis, 327336

Rémy JC, Hébert J (1977) Le devenir des engrais azotés dans le sol. CR Acad Agric Fr 63, 700-710

Sarniguet A (1990) Réceptivité des sols au piétinéchaudage du blé : influence des rotations et de la fertilisation azotée en relation avec certains facteurs physicochimiques et les peuplements de Pseudomonas fluorescents. Thèse doctorat $n^{\circ} 1212$, univ Paris XI-Orsay, $98 \mathrm{p}$

Schrödter H, Fehrmann H (1971a) Ökologische Untersuchungen zur Epidemiologie von Cercosporella herpotrichoides, II. Die Abhängigkeit des Infektionserfolges von einzelnen meteorologischen Faktoren. Phytopathol Z 71, 97-112

Schrödter H, Fehrmann H (1971b) Ökologische Untersuchungen zur Epidemiologie von Cercosporella herpotrichoides. III. Die relative Bedeutung der meteorologischen Parameter und die komplexe Wirkung ihrer Konstellationen auf den Infektionserfolg. Phytopathol Z 71, 203-222

Schulz H, Bodker L, Nistrup Jorgensen L, Kristensen K (1990) Influence of different cultural practices on distribution and incidence of eyespot (Pseudocercosporella herpotrichoides) in winter rye and winter wheat. Tidsskr Planteavl 94, 211-221

Scott PR (1970) Phialophora radiciola, an avirulant parasite of wheat and grass roots. Trans $\mathrm{Br} M y c o l$ Soc 55, 163-167

Sekerkova M, Bojnansky V, Turcany J (1982) Vpleyv zastupenia obilnin $v$ osevnych postupoch na vyskyt huby Cercosporella herpotrichoides a na urodnost ozimnef psenice (Effect of cereal proportions in crop rotations on the occurrence of the fungus Cercosporella herpotrichoides and on winter wheat yields). Sb uvtiz, Ochr Rost/ 18(1), 21-26

Shipton PJ (1972) Take-all in spring sown cereals under continuous cultivation, disease progress and decline in relation to crop succession and nitrogen. Ann Appl Biol 71, 33-46

Shipton PJ (1981) Saprophytic survival between susceptible crops. In : Biology and control of take-all (MJ Asher, PJ Shipton, eds), Academic Press, London, UK, 295-316

Slope DB, Etheridge J (1971) Grain yield and incidence of take-all (Ophiobolus graminis Sacc) in wheat grown in different crop sequences. Ann Appl Biol $67,13-22$

Slope DB, Prew RD, Gutteridge RJ, Etheridge J (1979) Take-all Gaeumannomyces graminis var tritici and the yield of wheat grown after ley and arable rotations in relation to the occurrence of Phialophora radiciola var graminicola. J Agric Sci, London 93, 377-389 
Steinbrenner K, Höflich G (1980) Bedeutung bodenbürtiger Getreidefußkrankheitserreger in spezialisierten Getreidefruchtfolgen und Möglichkeiten zu ihrer Bekämpfung. Tag ber, Akad Landwirtsch wiss dtsch demokr Repub 181, 29-36

Steinbrenner K, Höflich G (1984) Einfluß acker- und pflanzenbaulicher Maßnahmen auf den Befall des Getreides durch Pseudocercosporella herpotrichoides (Fron) Deighton und Gaeumannomyces graminis (Sacc) Arx et Olivier. Arch Phytopathol u Pflanzesch 20, 469-486

Steinbrenner K, Obenauf U (1986) Untersuchungen zum Einfluß der Vorfrucht und Vorvorfrucht auf den Ertrag von Wintergetreidearten und den Befall durch Gaeumannomyces graminis. Arch AckerPflanzenbau Bodenkd 30, 773-779

Steinbrenner K, Grabert D, Roth R, Obenauf U (1990) Fruchtfolgegestaltung - eine grundlegende
Maßnahme des Umweltschutzes. Nachrichtenbl Pflanzenschutz 44, 132-141

Sturz AV, Bernier CC (1989) Influence of crop rotations on winter wheat growth and yield in relation to the dynamics of pathogenic crown and root rot fungal complexes. Can J Plant Pathol 11, 114-121

Van der Hoeven EP, Bollen GJ (1980) Effect of benomyl on soil fungi associated with rye. Effect on the incidence of sharp eyes spot caused by Rhizoctonia cerealis. Neth J Plant Pathol 86, 163-180

Wehner F, Kammel M, Adam L (1988) Untersuchungen zum Auftreten der Halmbruchkrankheit in Getreide bei unterschiedlichen Vorfrüchten in der AgrarIndustrie-Vereinigung Berlstedt. Nachrichtenbl Pflanzenschutz 42(2), 122-125

Wehrle VM, Ogilvie L (1955) Effect of ley grasses on the carry-over of take-all. Plant Pathol 4, 111-113 